Supporting Information

\title{
FTIR Photoacoustic and ATR Spectroscopies of Soils with Aggregate Size Fractionation by Dry Sieving
}

Petr K. Krivoshein, ${ }^{1}$ Dmitry S. Volkov, ${ }^{1,2}$ Olga B. Rogova, ${ }^{2}$ and Mikhail A. Proskurnin* ${ }^{1}$

1. Chemistry Department, M.V. Lomonosov Moscow State University, Leninskie Gory, 1-3, GSP-1, Moscow, 119991, Russia

2. Department of Chemistry and Physical Chemistry of Soils, V.V. Dokuchaev Soil Science Institute, Pyzhevsky per., 7/2, Moscow 119017, Russia 

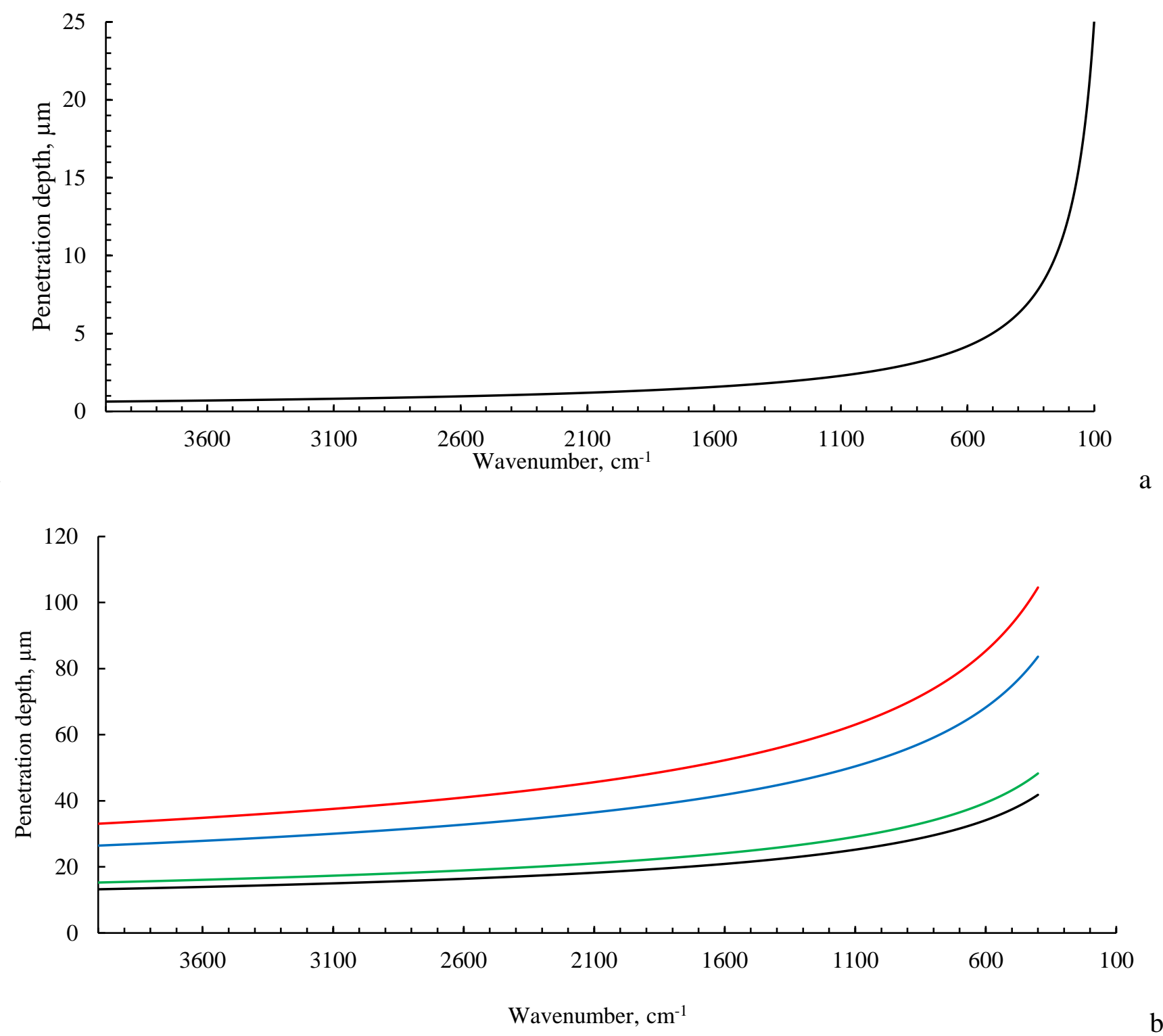

Figure S1. Dependence of the radiation penetration depth in (a) ATR-FTIR spectrometry (single reflection, diamond, $45^{\circ}$ ) for quartz and (b) FTIR-PAS spectrometry for chernozem soils, interferometer modulation frequencies: red, $1.6 \mathrm{kHz}$, blue $2.5 \mathrm{kHz}$, green, $7.5 \mathrm{kHz}$, black, $10.0 \mathrm{kHz}$. 

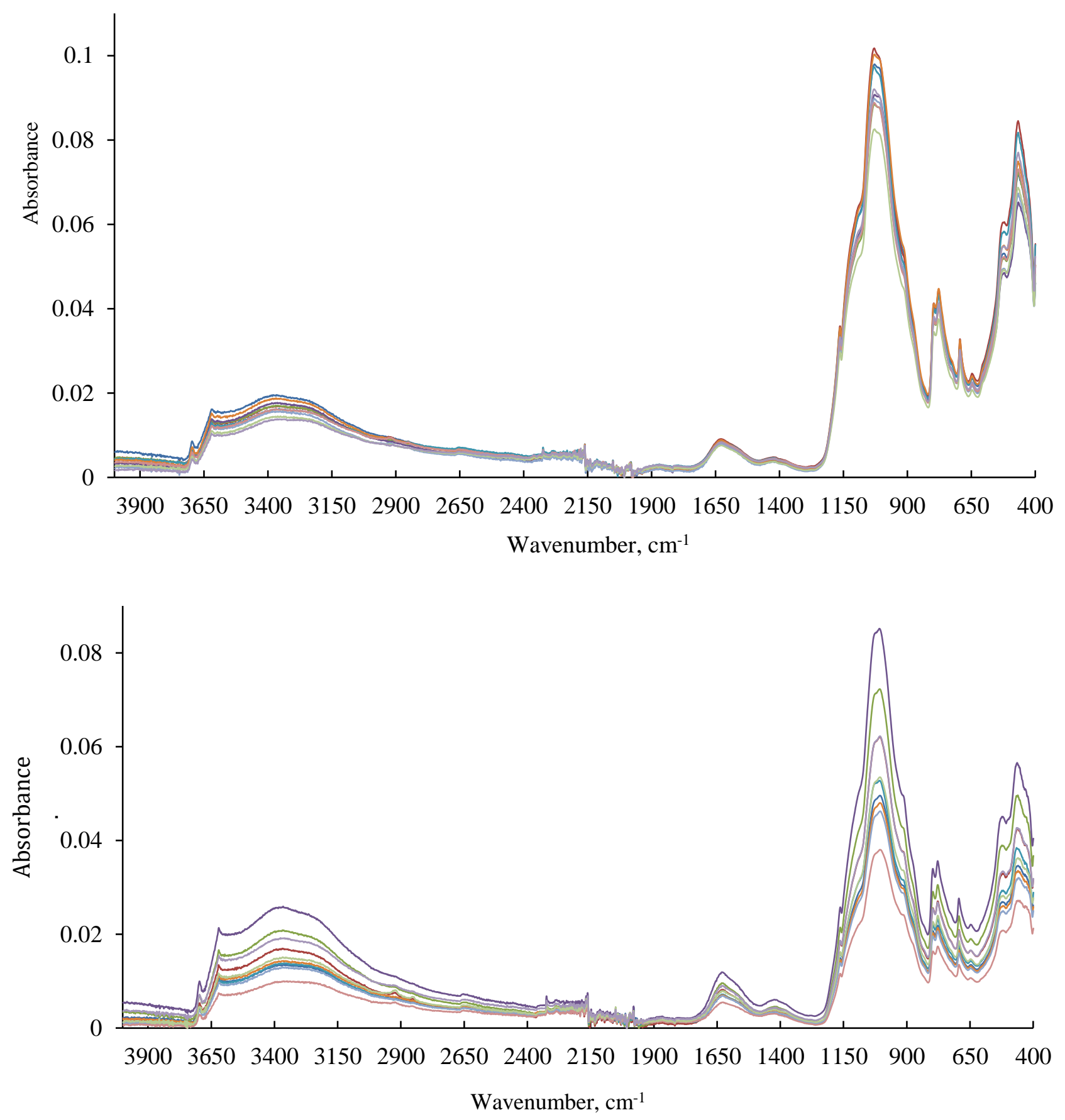

b

Figure S2. ATR-FTIR spectra of shelterbelt chernozem soil for fractions $<20 \mu \mathrm{m}$ (a) and 90-100 $\mu \mathrm{m}$ (b) for assessing reproducibility. 


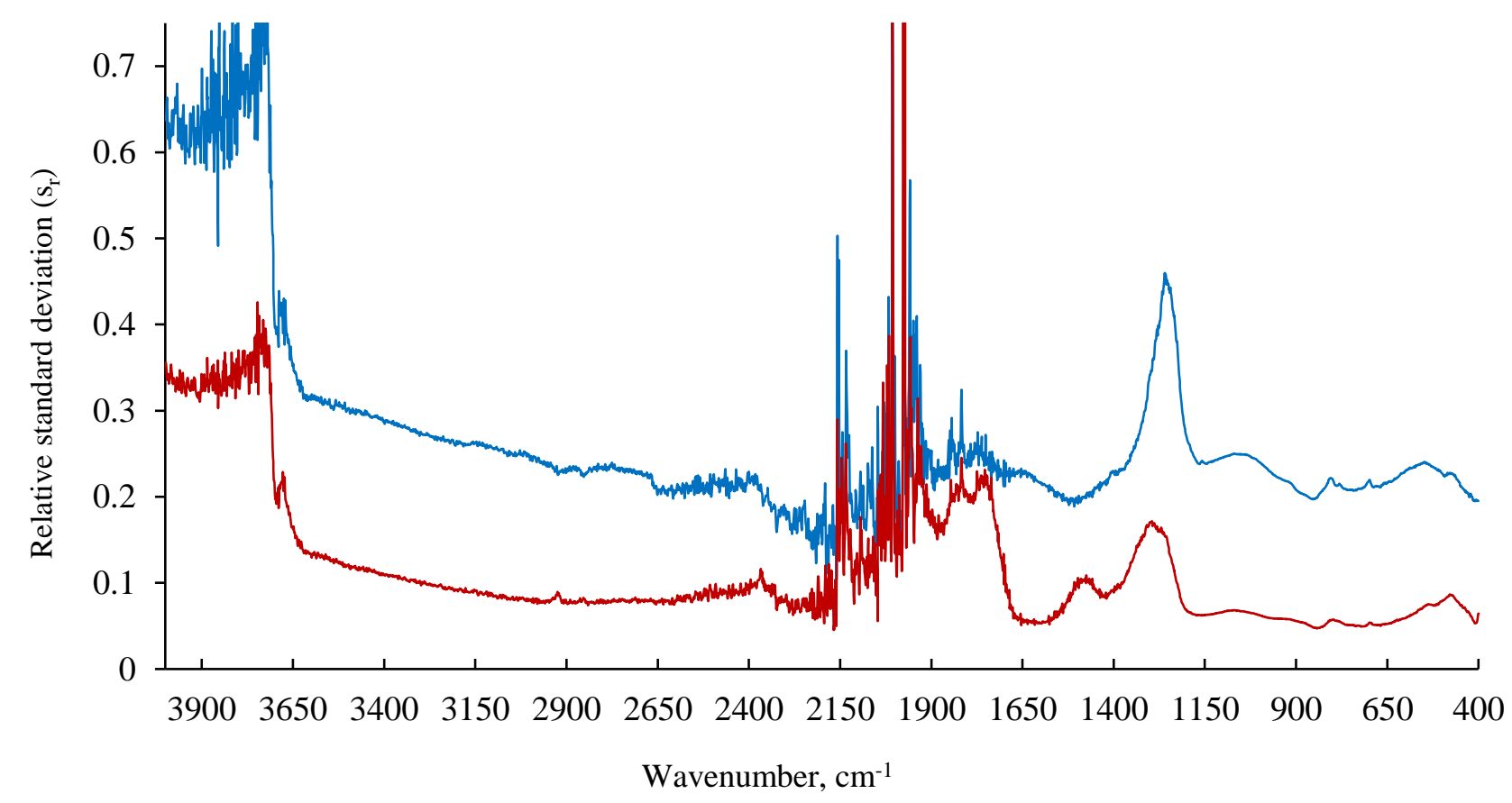

a

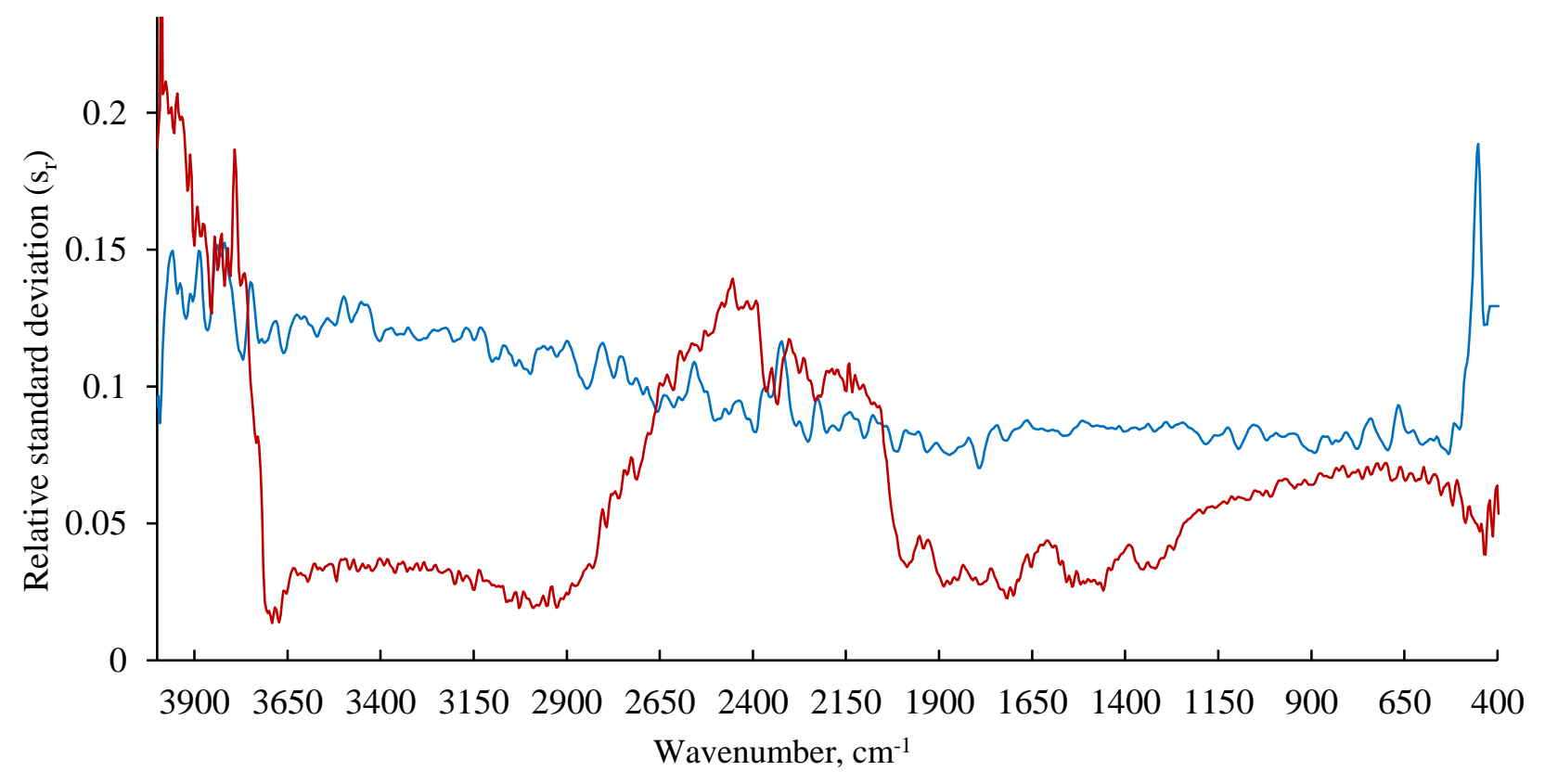

$\mathrm{b}$

Figure S3. Dependence of the relative standard deviation on the wavenumber for ATR-FTIR (a) and FTIR-PAS spectra (b, interferometer modulation frequency, $1.6 \mathrm{kHz}$ ) of shelterbelt chernozem soil for fractions below $20 \mu \mathrm{m}$ (red lines) and 90-100 $\mu \mathrm{m}$ (blue lines). The spectra were handled without additional processing. 


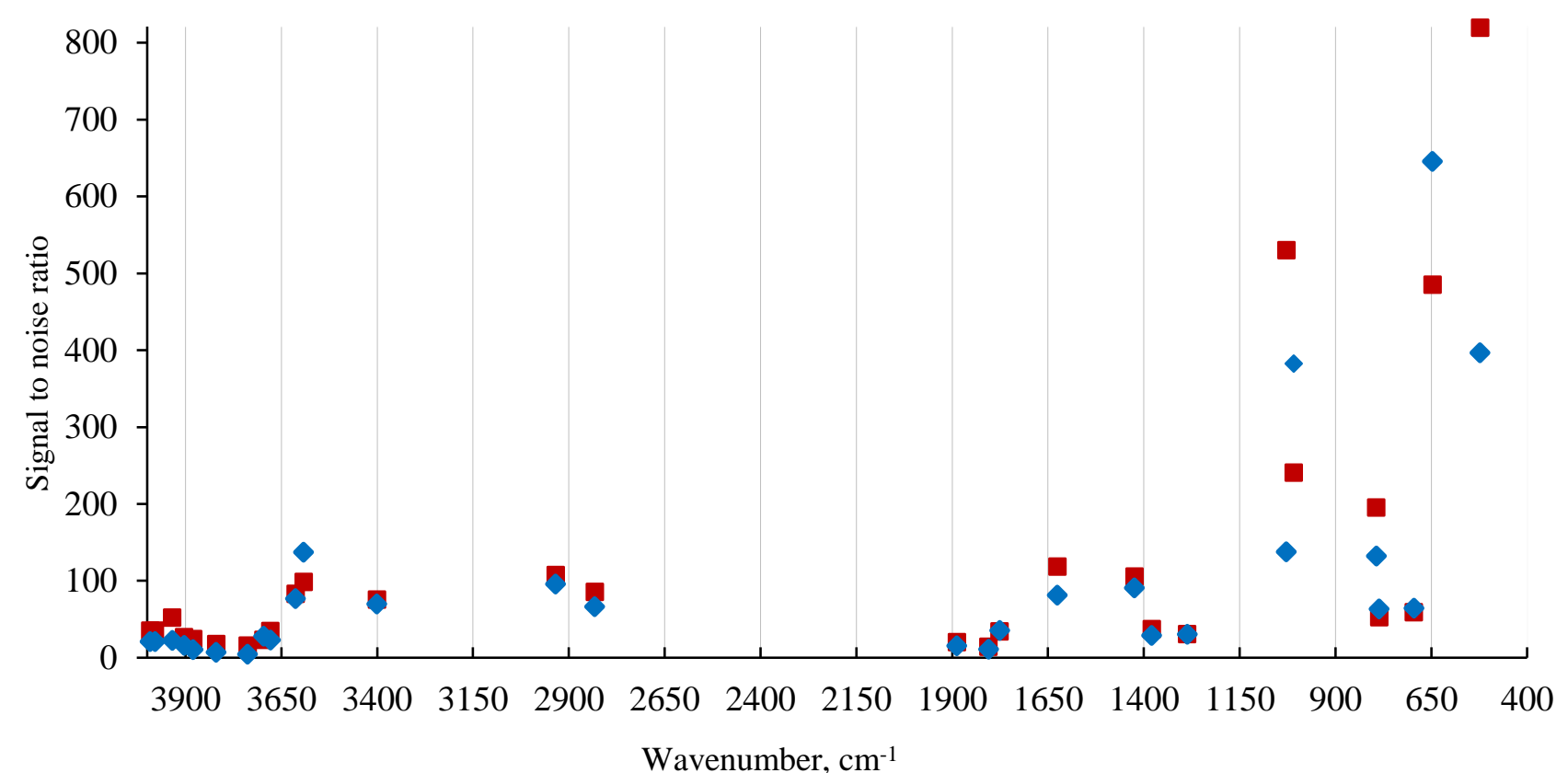

Wavenumber, $\mathrm{cm}^{-1}$

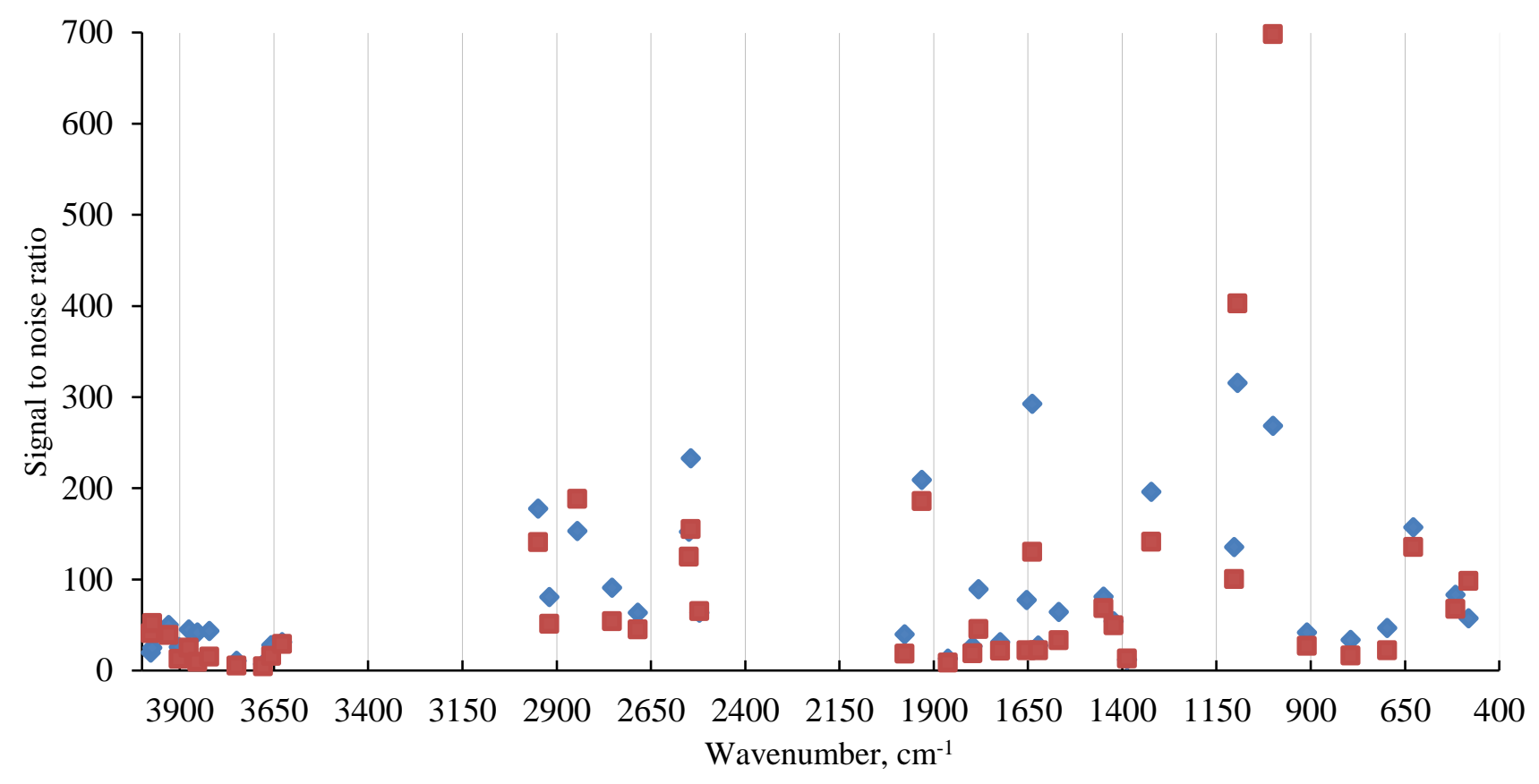

b

Figure S4. Comparison of the averaged signal-to-noise values for the most intense absorption bands for ATR-FTIR (a) and FTIR-PAS spectra (interferometer modulation frequency, $1.6 \mathrm{kHz}$ ) (b) of shelterbelt chernozem soil for fractions below $20 \mu \mathrm{m}$ (red squares) and 90-100 $\mu \mathrm{m}$ (blue rhombi). 
Table S1. Integral areas of ATR-FTIR bands of bare fallow chernozem soil

\begin{tabular}{|c|c|c|c|c|c|c|c|c|c|c|c|c|c|}
\hline \multirow[b]{2}{*}{$\begin{array}{c}\text { Band center, } \\
\mathrm{cm}^{-1}\end{array}$} & \multirow[b]{2}{*}{$\begin{array}{l}\text { High-wave boundary, } \\
\qquad \mathrm{cm}^{-1}\end{array}$} & \multirow[b]{2}{*}{$\begin{array}{l}\text { Low-wave boundary, } \\
\qquad \mathrm{cm}^{-1}\end{array}$} & \multicolumn{11}{|c|}{ Integral area } \\
\hline & & & $\begin{array}{l}<20 \\
\mu \mathrm{m}\end{array}$ & $\begin{array}{c}20-30 \\
\mu \mathrm{m}\end{array}$ & $\begin{array}{c}50-63 \\
\mu \mathrm{m}\end{array}$ & $\begin{array}{c}63-71 \\
\mu \mathrm{m}\end{array}$ & $\begin{array}{c}80-90 \\
\mu \mathrm{m}\end{array}$ & $\begin{array}{c}100-250 \\
\mu \mathrm{m}\end{array}$ & $\begin{array}{c}250-500 \\
\mu \mathrm{m}\end{array}$ & $\begin{array}{c}500-1000 \\
\mu \mathrm{m}\end{array}$ & $\begin{array}{l}1-2 \\
\mathrm{~mm}\end{array}$ & $\begin{array}{l}2-5 \\
\mathrm{~mm}\end{array}$ & $\begin{array}{l}>5 \\
\mathrm{~mm}\end{array}$ \\
\hline 3693 & 3704 & 3683 & 0.007 & 0.010 & 0.017 & 0.015 & 0.019 & 0.010 & 0.013 & 0.011 & 0.011 & 0.001 & 0.000 \\
\hline 3619 & 3636 & 3601 & 0.026 & 0.016 & 0.023 & 0.020 & 0.027 & 0.048 & 0.033 & 0.042 & 0.044 & 0.020 & 0.006 \\
\hline 3565 & 3574 & 3555 & 0.003 & 0.003 & 0.000 & 0.004 & 0.004 & 0.000 & 0.002 & 0.001 & 0.002 & 0.000 & 0.002 \\
\hline 2928 & 2946 & 2910 & 0.000 & 0.000 & 0.000 & 0.000 & 0.016 & 0.000 & 0.003 & 0.000 & 0.000 & 0.009 & 0.000 \\
\hline 2847 & 2864 & 2831 & 0.000 & 0.001 & 0.000 & 0.000 & 0.008 & 0.002 & 0.000 & 0.000 & 0.000 & 0.000 & 0.000 \\
\hline 1620 & 1744 & 1497 & 1.034 & 0.697 & 0.798 & 1.085 & 1.194 & 1.242 & 1.296 & 1.479 & 1.346 & 1.050 & 0.755 \\
\hline 1405 & 1467 & 1344 & 0.092 & 0.060 & 0.089 & 0.113 & 0.125 & 0.129 & 0.125 & 0.146 & 0.131 & 0.109 & 0.082 \\
\hline 1165 & 1176 & 1154 & 0.076 & 0.060 & 0.041 & 0.037 & 0.040 & 0.041 & 0.047 & 0.052 & 0.055 & 0.041 & 0.035 \\
\hline 1111 & 1141 & 1082 & 0.238 & 0.152 & 0.090 & 0.100 & 0.110 & 0.124 & 0.155 & 0.165 & 0.177 & 0.171 & 0.142 \\
\hline 1037 & 1060 & 1014 & 0.307 & 0.219 & 0.164 & 0.181 & 0.163 & 0.196 & 0.235 & 0.258 & 0.276 & 0.263 & 0.222 \\
\hline 989 & 1010 & 967 & 0.304 & 0.183 & 0.159 & 0.193 & 0.215 & 0.241 & 0.290 & 0.331 & 0.308 & 0.265 & 0.192 \\
\hline 780 & 823 & 736 & 2.576 & 1.664 & 1.094 & 1.221 & 1.187 & 1.300 & 1.486 & 1.710 & 1.751 & 1.364 & 1.117 \\
\hline 690 & 709 & 672 & 0.384 & 0.274 & 0.179 & 0.209 & 0.196 & 0.321 & 0.241 & 0.273 & 0.282 & 0.320 & 0.175 \\
\hline 644 & 663 & 624 & 0.218 & 0.068 & 0.014 & 0.006 & 0.024 & 0.165 & 0.228 & 0.243 & 0.271 & 0.132 & 0.076 \\
\hline 450 & 494 & 405 & 5.519 & 3.924 & 2.456 & 2.558 & 2.185 & 2.648 & 3.210 & 3.504 & 3.725 & 3.404 & 2.958 \\
\hline 389 & 396 & 383 & 0.156 & 0.098 & 0.064 & 0.066 & 0.069 & 0.083 & 0.094 & 0.100 & 0.104 & 0.070 & 0.063 \\
\hline 353 & 367 & 340 & 0.176 & 0.123 & 0.068 & 0.082 & 0.063 & 0.035 & 0.043 & 0.052 & 0.046 & 0.000 & 0.016 \\
\hline 321 & 338 & 304 & 0.180 & 0.124 & 0.044 & 0.099 & 0.038 & 0.057 & 0.078 & 0.072 & 0.093 & 0.056 & 0.057 \\
\hline 254 & 272 & 235 & 0.698 & 0.540 & 0.246 & 0.226 & 0.201 & 0.252 & 0.286 & 0.307 & 0.329 & 0.285 & 0.259 \\
\hline
\end{tabular}


Table S2. Integral areas of ATR-FTIR bands of native steppe chernozem soil

\begin{tabular}{|c|c|c|c|c|c|c|c|c|c|c|c|c|c|}
\hline \multirow[b]{2}{*}{$\begin{array}{c}\text { Band center, } \\
\mathrm{cm}^{-1}\end{array}$} & \multirow[b]{2}{*}{$\begin{array}{l}\text { High-wave boundary, } \\
\qquad \mathrm{cm}^{-1}\end{array}$} & \multirow[b]{2}{*}{$\begin{array}{l}\text { Low-wave boundary, } \\
\qquad \mathrm{cm}^{-1}\end{array}$} & \multicolumn{11}{|c|}{ Integral area } \\
\hline & & & $\begin{array}{l}<20 \\
\mu \mathrm{m}\end{array}$ & $\begin{array}{l}20-30 \\
\mu \mathrm{m}\end{array}$ & $\begin{array}{c}50-63 \\
\mu \mathrm{m}\end{array}$ & $\begin{array}{c}63-71 \\
\mu \mathrm{m}\end{array}$ & $\begin{array}{c}80-90 \\
\mu \mathrm{m}\end{array}$ & $\begin{array}{c}100-250 \\
\mu \mathrm{m}\end{array}$ & $\begin{array}{c}250-500 \\
\mu \mathrm{m}\end{array}$ & $\begin{array}{c}500-1000 \\
\mu \mathrm{m}\end{array}$ & $\begin{array}{l}1-2 \\
\mathrm{~mm}\end{array}$ & $\begin{array}{l}2-5 \\
\mathrm{~mm}\end{array}$ & $\begin{array}{l}>5 \\
\mathrm{~mm}\end{array}$ \\
\hline 3693 & 3704 & 3683 & 0.002 & 0.008 & 0.007 & 0.004 & 0.001 & 0.011 & 0.014 & 0.011 & 0.004 & 0.010 & 0.000 \\
\hline 3619 & 3636 & 3601 & -0.001 & 0.004 & 0.015 & 0.020 & 0.001 & 0.026 & 0.041 & 0.017 & 0.015 & 0.033 & 0.008 \\
\hline 3565 & 3574 & 3555 & 0.004 & 0.003 & 0.004 & 0.005 & 0.002 & 0.002 & 0.005 & 0.000 & 0.005 & 0.001 & 0.001 \\
\hline 2928 & 2946 & 2910 & 0.001 & 0.007 & 0.000 & 0.014 & 0.024 & 0.000 & 0.000 & 0.003 & 0.000 & 0.000 & 0.019 \\
\hline 2847 & 2864 & 2831 & 0.000 & 0.002 & 0.002 & 0.017 & 0.018 & 0.002 & 0.001 & 0.007 & 0.004 & 0.000 & 0.015 \\
\hline 1620 & 1744 & 1497 & 0.756 & 0.741 & 1.065 & 1.513 & 1.060 & 1.358 & 1.541 & 1.088 & 0.935 & 1.176 & 1.502 \\
\hline 1405 & 1467 & 1344 & 0.062 & 0.072 & 0.128 & 0.173 & 0.126 & 0.151 & 0.172 & 0.126 & 0.105 & 0.125 & 0.175 \\
\hline 1165 & 1176 & 1154 & 0.066 & 0.058 & 0.037 & 0.038 & 0.030 & 0.035 & 0.037 & 0.031 & 0.030 & 0.041 & 0.042 \\
\hline 1111 & 1141 & 1082 & 0.229 & 0.166 & 0.095 & 0.091 & 0.075 & 0.101 & 0.110 & 0.102 & 0.094 & 0.142 & 0.124 \\
\hline 1037 & 1060 & 1014 & 0.236 & 0.190 & 0.141 & 0.145 & 0.116 & 0.151 & 0.160 & 0.154 & 0.137 & 0.209 & 0.180 \\
\hline 989 & 1010 & 967 & 0.173 & 0.147 & 0.159 & 0.203 & 0.151 & 0.192 & 0.216 & 0.173 & 0.148 & 0.232 & 0.194 \\
\hline 780 & 823 & 736 & 2.041 & 1.524 & 0.971 & 1.061 & 0.830 & 1.053 & 1.147 & 0.952 & 0.895 & 1.257 & 1.269 \\
\hline 690 & 709 & 672 & 0.289 & 0.260 & 0.160 & 0.176 & 0.133 & 0.167 & 0.178 & 0.145 & 0.134 & 0.194 & 0.186 \\
\hline 644 & 663 & 624 & 0.344 & 0.214 & 0.129 & 0.009 & 0.051 & 0.000 & 0.043 & 0.000 & 0.000 & 0.028 & 0.000 \\
\hline 450 & 494 & 405 & 4.364 & 3.455 & 2.175 & 2.070 & 1.709 & 2.115 & 2.179 & 2.114 & 1.943 & 3.090 & 2.586 \\
\hline 389 & 396 & 383 & 0.107 & 0.075 & 0.062 & 0.064 & 0.048 & 0.056 & 0.061 & 0.053 & 0.044 & 0.075 & 0.056 \\
\hline 353 & 367 & 340 & 0.145 & 0.089 & 0.028 & 0.034 & 0.024 & 0.039 & 0.044 & 0.042 & 0.020 & 0.034 & 0.037 \\
\hline 321 & 338 & 304 & 0.184 & 0.087 & 0.044 & 0.030 & 0.034 & 0.070 & 0.050 & 0.058 & 0.059 & 0.096 & 0.077 \\
\hline 254 & 272 & 235 & 0.633 & 0.423 & 0.160 & 0.172 & 0.143 & 0.194 & 0.222 & 0.223 & 0.196 & 0.298 & 0.235 \\
\hline
\end{tabular}


Table S3. Integral areas of ATR-FTIR bands of shelterbelt chernozem soil

\begin{tabular}{|c|c|c|c|c|c|c|c|c|c|c|c|c|c|}
\hline \multirow[b]{2}{*}{$\begin{array}{l}\text { Band center, } \\
\qquad \mathrm{cm}^{-1}\end{array}$} & \multirow[b]{2}{*}{$\begin{array}{l}\text { High-wave boundary, } \\
\qquad \mathrm{cm}^{-1}\end{array}$} & \multirow[b]{2}{*}{$\begin{array}{l}\text { Low-wave boundary, } \\
\qquad \mathrm{cm}^{-1}\end{array}$} & \multicolumn{11}{|c|}{ Integral area } \\
\hline & & & $\begin{array}{l}<20 \\
\mu \mathrm{m}\end{array}$ & $\begin{array}{c}20-30 \\
\mu \mathrm{m}\end{array}$ & $\begin{array}{c}50-63 \\
\mu \mathrm{m}\end{array}$ & $\begin{array}{c}63-71 \\
\mu \mathrm{m}\end{array}$ & $\begin{array}{c}80-90 \\
\mu \mathrm{m}\end{array}$ & $\begin{array}{c}100-250 \\
\mu \mathrm{m}\end{array}$ & $\begin{array}{c}250-500 \\
\mu \mathrm{m}\end{array}$ & $\begin{array}{c}500-1000 \\
\mu \mathrm{m}\end{array}$ & $\begin{array}{l}1-2 \\
\mathrm{~mm}\end{array}$ & $\begin{array}{l}2-5 \\
\mathrm{~mm}\end{array}$ & $\begin{array}{l}>5 \\
\mathrm{~mm}\end{array}$ \\
\hline 3693 & 3704 & 3683 & 0.011 & 0.000 & 0.002 & 0.004 & 0.015 & 0.016 & 0.001 & 0.013 & 0.010 & 0.010 & 0.021 \\
\hline 3619 & 3636 & 3601 & 0.019 & 0.001 & 0.020 & 0.020 & 0.011 & 0.046 & 0.029 & 0.034 & 0.032 & 0.023 & 0.027 \\
\hline 3565 & 3574 & 3555 & 0.003 & 0.003 & 0.002 & 0.000 & 0.000 & 0.000 & 0.000 & 0.000 & 0.002 & 0.000 & 0.000 \\
\hline 2928 & 2946 & 2910 & 0.003 & 0.000 & 0.000 & 0.005 & 0.010 & 0.002 & 0.000 & 0.000 & 0.004 & 0.001 & 0.007 \\
\hline 2847 & 2864 & 2831 & 0.001 & 0.003 & 0.000 & 0.003 & 0.007 & 0.007 & 0.003 & 0.005 & 0.005 & 0.006 & 0.007 \\
\hline 1620 & 1744 & 1497 & 1.323 & 0.960 & 1.023 & 1.046 & 0.933 & 1.482 & 1.167 & 1.120 & 1.512 & 1.267 & 1.614 \\
\hline 1405 & 1467 & 1344 & 0.141 & 0.111 & 0.122 & 0.117 & 0.092 & 0.173 & 0.133 & 0.135 & 0.179 & 0.150 & 0.190 \\
\hline 1165 & 1176 & 1154 & 0.067 & 0.054 & 0.035 & 0.032 & 0.025 & 0.037 & 0.033 & 0.030 & 0.047 & 0.037 & 0.047 \\
\hline 1111 & 1141 & 1082 & 0.261 & 0.181 & 0.102 & 0.082 & 0.084 & 0.127 & 0.117 & 0.111 & 0.158 & 0.147 & 0.136 \\
\hline 1037 & 1060 & 1014 & 0.350 & 0.254 & 0.157 & 0.133 & 0.122 & 0.195 & 0.162 & 0.159 & 0.233 & 0.206 & 0.192 \\
\hline 989 & 1010 & 967 & 0.337 & 0.191 & 0.164 & 0.153 & 0.134 & 0.229 & 0.183 & 0.178 & 0.272 & 0.217 & 0.230 \\
\hline 780 & 823 & 736 & 2.395 & 1.542 & 0.921 & 0.850 & 0.726 & 1.161 & 1.029 & 0.927 & 1.495 & 1.226 & 1.434 \\
\hline 690 & 709 & 672 & 0.340 & 0.231 & 0.279 & 0.273 & 0.121 & 0.183 & 0.163 & 0.145 & 0.221 & 0.179 & 0.319 \\
\hline 644 & 663 & 624 & 0.242 & 0.094 & 0.170 & 0.179 & 0.201 & 0.131 & 0.157 & 0.095 & 0.268 & 0.223 & 0.294 \\
\hline 450 & 494 & 405 & 5.827 & 3.839 & 2.305 & 1.910 & 1.609 & 2.632 & 2.178 & 2.150 & 3.556 & 2.797 & 2.876 \\
\hline 389 & 396 & 383 & 0.142 & 0.074 & 0.057 & 0.054 & 0.046 & 0.070 & 0.056 & 0.041 & 0.083 & 0.060 & 0.069 \\
\hline 353 & 367 & 340 & 0.113 & 0.059 & 0.025 & 0.013 & 0.025 & 0.031 & 0.012 & 0.003 & 0.023 & 0.000 & 0.024 \\
\hline 321 & 338 & 304 & 0.188 & 0.088 & 0.046 & 0.041 & 0.039 & 0.062 & 0.039 & 0.022 & 0.068 & 0.025 & 0.061 \\
\hline 254 & 272 & 235 & 0.686 & 0.428 & 0.208 & 0.154 & 0.158 & 0.253 & 0.216 & 0.178 & 0.326 & 0.217 & 0.226 \\
\hline
\end{tabular}


Table S4. Integral areas of ATR-FTIR bands of cropland chernozem soil

\begin{tabular}{|c|c|c|c|c|c|c|c|c|c|c|c|c|c|}
\hline \multirow[b]{2}{*}{$\begin{array}{l}\text { Band center, } \\
\qquad \mathrm{cm}^{-1}\end{array}$} & \multirow[b]{2}{*}{$\begin{array}{l}\text { High-wave boundary, } \\
\qquad \mathrm{cm}^{-1}\end{array}$} & \multirow[b]{2}{*}{$\begin{array}{l}\text { Low-wave boundary, } \\
\qquad \mathrm{cm}^{-1}\end{array}$} & \multicolumn{11}{|c|}{ Integral area } \\
\hline & & & $\begin{array}{l}<20 \\
\mu \mathrm{m}\end{array}$ & $\begin{array}{c}20-30 \\
\mu \mathrm{m}\end{array}$ & $\begin{array}{c}50-63 \\
\mu \mathrm{m}\end{array}$ & $\begin{array}{c}63-71 \\
\mu \mathrm{m}\end{array}$ & $\begin{array}{c}80-90 \\
\mu \mathrm{m}\end{array}$ & $\begin{array}{c}100-250 \\
\mu \mathrm{m}\end{array}$ & $\begin{array}{c}250-500 \\
\mu \mathrm{m}\end{array}$ & $\begin{array}{c}500-1000 \\
\mu \mathrm{m}\end{array}$ & $\begin{array}{l}1-2 \\
\mathrm{~mm}\end{array}$ & $\begin{array}{l}2-5 \\
\mathrm{~mm}\end{array}$ & $\begin{array}{l}>5 \\
\mathrm{~mm}\end{array}$ \\
\hline 3693 & 3704 & 3683 & 0.010 & 0.000 & 0.009 & 0.013 & 0.019 & 0.017 & 0.021 & 0.021 & 0.017 & 0.028 & 0.007 \\
\hline 3619 & 3636 & 3601 & 0.027 & 0.018 & 0.010 & 0.019 & 0.029 & 0.048 & 0.056 & 0.042 & 0.035 & 0.049 & 0.023 \\
\hline 3565 & 3574 & 3555 & -0.002 & 0.000 & 0.005 & 0.004 & 0.004 & 0.000 & 0.002 & 0.005 & 0.000 & 0.000 & 0.001 \\
\hline 2928 & 2946 & 2910 & 0.003 & 0.006 & 0.000 & 0.000 & 0.000 & 0.000 & 0.001 & 0.033 & 0.000 & 0.000 & 0.000 \\
\hline 2847 & 2864 & 2831 & 0.002 & 0.003 & 0.000 & 0.001 & 0.000 & 0.000 & 0.000 & 0.023 & 0.000 & 0.001 & 0.000 \\
\hline 1620 & 1744 & 1497 & 1.084 & 0.898 & 0.871 & 1.024 & 1.380 & 1.339 & 1.962 & 1.624 & 1.601 & 1.854 & 0.435 \\
\hline 1405 & 1467 & 1344 & 0.113 & 0.000 & 0.105 & 0.117 & 0.169 & 0.180 & 0.217 & 0.174 & 0.185 & 0.220 & 0.226 \\
\hline 1165 & 1176 & 1154 & 0.069 & 0.061 & 0.044 & 0.040 & 0.046 & 0.046 & 0.056 & 0.057 & 0.047 & 0.064 & 0.035 \\
\hline 1111 & 1141 & 1082 & 0.202 & 0.131 & 0.100 & 0.097 & 0.118 & 0.121 & 0.161 & 0.147 & 0.139 & 0.177 & 0.118 \\
\hline 1037 & 1060 & 1014 & 0.293 & 0.226 & 0.180 & 0.159 & 0.188 & 0.196 & 0.240 & 0.239 & 0.216 & 0.280 & 0.203 \\
\hline 989 & 1010 & 967 & 0.293 & 0.196 & 0.170 & 0.190 & 0.251 & 0.263 & 0.356 & 0.330 & 0.310 & 0.412 & 0.216 \\
\hline 780 & 823 & 736 & 2.302 & 1.700 & 1.160 & 1.066 & 1.344 & 1.366 & 1.727 & 1.721 & 1.442 & 1.987 & 1.055 \\
\hline 690 & 709 & 672 & 0.360 & 0.262 & 0.183 & 0.173 & 0.220 & 0.219 & 0.282 & 0.279 & 0.222 & 0.311 & 0.161 \\
\hline 644 & 663 & 624 & 0.161 & 0.208 & 0.303 & 0.156 & 0.206 & 0.223 & 0.182 & 0.234 & 0.301 & 0.268 & 0.178 \\
\hline 450 & 494 & 405 & 4.679 & 3.712 & 2.582 & 2.125 & 2.627 & 2.584 & 3.136 & 3.284 & 2.742 & 3.723 & 2.411 \\
\hline 389 & 396 & 383 & 0.134 & 0.091 & 0.067 & 0.063 & 0.082 & 0.081 & 0.098 & 0.102 & 0.088 & 0.111 & 0.054 \\
\hline 353 & 367 & 340 & 0.155 & 0.087 & 0.041 & 0.033 & 0.050 & 0.046 & 0.056 & 0.044 & 0.022 & 0.042 & 0.000 \\
\hline 321 & 338 & 304 & 0.177 & 0.086 & 0.044 & 0.040 & 0.063 & 0.052 & 0.052 & 0.053 & 0.041 & 0.051 & 0.032 \\
\hline 254 & 272 & 235 & 0.588 & 0.377 & 0.230 & 0.198 & 0.230 & 0.216 & 0.246 & 0.253 & 0.191 & 0.302 & 0.191 \\
\hline
\end{tabular}


Table S5. Integral areas of ATR-FTIR bands of sod-podzolic soil

\begin{tabular}{|c|c|c|c|c|c|c|c|c|c|c|c|c|c|}
\hline \multirow[b]{2}{*}{$\begin{array}{c}\text { Band center, } \\
\qquad \mathrm{cm}^{-1}\end{array}$} & \multirow[b]{2}{*}{$\begin{array}{l}\text { High-wave boundary, } \\
\qquad \mathrm{cm}^{-1}\end{array}$} & \multirow[b]{2}{*}{$\begin{array}{l}\text { Low-wave boundary, } \\
\qquad \mathrm{cm}^{-1}\end{array}$} & \multicolumn{11}{|c|}{ Integral area } \\
\hline & & & $\begin{array}{l}<20 \\
\mu \mathrm{m}\end{array}$ & $\begin{array}{c}20-30 \\
\mu \mathrm{m}\end{array}$ & $\begin{array}{c}50-63 \\
\mu \mathrm{m}\end{array}$ & $\begin{array}{c}63-71 \\
\mu \mathrm{m}\end{array}$ & $\begin{array}{c}80-90 \\
\mu \mathrm{m}\end{array}$ & $\begin{array}{c}100-250 \\
\mu \mathrm{m}\end{array}$ & $\begin{array}{c}250-500 \\
\mu \mathrm{m}\end{array}$ & $\begin{array}{c}500-1000 \\
\mu \mathrm{m}\end{array}$ & $\begin{array}{l}1-2 \\
\mathrm{~mm}\end{array}$ & $\begin{array}{l}2-5 \\
\mathrm{~mm}\end{array}$ & $\begin{array}{l}>5 \\
\mathrm{~mm}\end{array}$ \\
\hline 3693 & 3704 & 3683 & 0.010 & 0.009 & 0.008 & 0.013 & 0.007 & 0.008 & 0.011 & 0.014 & 0.009 & 0.005 & 0.009 \\
\hline 3619 & 3636 & 3601 & 0.015 & 0.012 & 0.009 & 0.018 & 0.009 & 0.005 & 0.013 & 0.020 & 0.014 & 0.005 & 0.014 \\
\hline 3565 & 3574 & 3555 & 0.000 & 0.000 & 0.000 & 0.000 & 0.000 & 0.000 & 0.000 & 0.000 & 0.000 & 0.000 & 0.000 \\
\hline 2928 & 2946 & 2910 & 0.006 & 0.005 & 0.003 & 0.016 & 0.002 & 0.004 & 0.012 & 0.014 & 0.011 & 0.007 & 0.010 \\
\hline 2847 & 2864 & 2831 & 0.004 & 0.003 & 0.003 & 0.009 & 0.002 & 0.002 & 0.006 & 0.007 & 0.005 & 0.003 & 0.006 \\
\hline 1620 & 1744 & 1497 & 0.491 & 0.406 & 0.352 & 0.607 & 0.342 & 0.360 & 0.513 & 0.632 & 0.469 & 0.192 & 0.672 \\
\hline 1405 & 1467 & 1344 & 0.032 & 0.031 & 0.038 & 0.056 & 0.041 & 0.040 & 0.052 & 0.060 & 0.042 & 0.019 & 0.065 \\
\hline 1165 & 1176 & 1154 & 0.083 & 0.068 & 0.041 & 0.053 & 0.031 & 0.048 & 0.049 & 0.058 & 0.050 & 0.046 & 0.074 \\
\hline 1111 & 1141 & 1082 & 0.327 & 0.241 & 0.136 & 0.159 & 0.113 & 0.204 & 0.155 & 0.152 & 0.143 & 0.183 & 0.205 \\
\hline 1037 & 1060 & 1014 & 0.361 & 0.255 & 0.152 & 0.190 & 0.125 & 0.218 & 0.189 & 0.199 & 0.180 & 0.179 & 0.277 \\
\hline 989 & 1010 & 967 & 0.287 & 0.206 & 0.143 & 0.232 & 0.133 & 0.176 & 0.207 & 0.251 & 0.173 & 0.131 & 0.255 \\
\hline 780 & 823 & 736 & 3.047 & 2.083 & 1.185 & 1.650 & 0.962 & 1.522 & 1.556 & 1.913 & 1.637 & 1.398 & 2.409 \\
\hline 690 & 709 & 672 & 0.400 & 0.294 & 0.168 & 0.237 & 0.138 & 0.206 & 0.221 & 0.273 & 0.226 & 0.187 & 0.332 \\
\hline 644 & 663 & 624 & 0.135 & 0.087 & 0.049 & 0.066 & 0.039 & 0.064 & 0.065 & 0.084 & 0.069 & 0.065 & 0.105 \\
\hline 450 & 494 & 405 & 5.941 & 4.057 & 2.200 & 2.897 & 1.783 & 3.206 & 2.718 & 2.961 & 2.700 & 2.855 & 4.806 \\
\hline
\end{tabular}

